\title{
Observation of anisotropic interlayer Raman modes in few-layer $\mathbf{R e S}_{2}$
}

\author{
Philipp Nagler, ${ }^{1}$ Gerd Plechinger, ${ }^{1}$ Christian Schüller, ${ }^{1}$ and Tobias Korn ${ }^{1, *}$ \\ ${ }^{1}$ Institut für Experimentelle und Angewandte Physik, \\ Universität Regensburg, D-93040 Regensburg, Germany
}

\begin{abstract}
$\mathrm{ReS}_{2}$ has recently emerged as a new member in the rapidly growing family of two-dimensional materials. Unlike $\mathrm{MoS}_{2}$ or $\mathrm{WSe}_{2}$, the optical and electrical properties of $\mathrm{ReS}_{2}$ are not isotropic due to the reduced symmetry of the crystal. Here, we present layer-dependent Raman measurements of $\mathrm{ReS}_{2}$ samples ranging from monolayers to ten layers in the ultralow frequency regime. We observe layer breathing and shear modes which allow for easy assignment of the number of layers. Polarization-dependent measurements give further insight into the crystal structure and reveal an energetic shift of the shear mode which stems from the in-plane anisotropy of the shear modulus in this material.
\end{abstract}

In the emerging field of two-dimensional (2D) materials science, reports of atomically thin $\operatorname{ReS}_{2} \frac{1}{1}$ have lately attracted increasing interest in the research community. $\mathrm{ReS}_{2}$ is a layered semiconductor with a bulk band gap of about $1.5 \mathrm{eV}$, where one monolayer (ML) consists of one lattice of rhenium atoms which is sandwiched between two lattices of sulfur atoms. Like in $\mathrm{MoS}_{2}$ or $\mathrm{WSe}_{2}$, the weak van der Waals (vdW) interaction between individual MLs enables the fabrication of atomically thin samples by mechanical exfoliation ${ }^{2}$. However, in contrast to many other transition metal dichalcogenides (TMDCs), $\mathrm{ReS}_{2}$ and $\mathrm{ReSe}_{2}$ do not crystallize in a $2 \mathrm{H}$ structure but in a distorted $1 \mathrm{~T}$ structure due to charge decoupling from an additional valence electron in the rhenium atoms ${ }^{113}$ which leads to a reduced crystal symmetry. Recently, it has also been proposed that $\operatorname{ReS}_{2}$ is not isostructural to $\mathrm{ReSe}_{2}$, but crystallizes in a different structure containing only a single $\mathrm{ReS}_{2}$ layer per unit cell ${ }^{4}$. The in-plane anisotropy of $\mathrm{ReS}_{2}$ has already been examined in electrical and optical studies on bulk samples in the past $t \frac{516}{6}$. Very recently, these experiments were extended to atomically thin $\mathrm{ReS}_{2}$ and have demonstrated the potential of the material for future ultra-thin optoelectronic devices ${ }^{7-10}$. These hopes are also nurtured by alternative production methods for atomically thin $\mathrm{ReS}_{2}$ such as chemical exfoliation 11 and CVD growth 12 .

Raman spectroscopy has been established as a powerful tool to study elementary excitations in layered 2D materials by optical means $13[14$. First Raman studies on atomically thin $\mathrm{ReS}_{2}$ in the high-frequency range $\left(>100 \mathrm{~cm}^{-1}\right)$ have already provided insight into the intralayer modes ${ }^{4}$ and their anisotropic behaviour ${ }^{15}$. However, in addition to the intralayer modes, the weak vdW coupling in layered crystal structures usually leads to the emergence of interlayer phonon modes which are typically very low in energy $\left(<50 \mathrm{~cm}^{-1}\right)$. Thereby, each layer oscillates rigidly as a whole unit which leads to two distinct features: layer shear modes (LSM), where the layers oscillate rigidly against each other in the plane of the crystal, and layer breathing modes (LBM), where the oscillation amplitude is perpendicular to the layer plane. Pioneered for graphene ${ }^{16}$, the study of interlayer phonon modes in atomically thin layered materials was soon extended to TMDCs such as $\mathrm{MoS}_{2} \frac{17 \mid 18}{}, \mathrm{WSe}_{2} \frac{19}{,}, \mathrm{WS}_{2}{ }^{20}$ and $\mathrm{MoTe}_{2} 21$. The observed interlayer phonon modes provide valuable information about the forces acting in the crystal and their layer-dependent behavior renders them a favorable tool for determining the thickness of the material. However, an investigation of the low-energetic interlayer modes of $\mathrm{ReS}_{2}$ is still lacking. It was also argued that for the proposed bulk crystal structure containing only a single $\mathrm{ReS}_{2}$ layer per unit cell ${ }^{4}$, the interlayer phonon modes should not be present at all in $\mathrm{ReS}_{2}$.

Here, we aim to clarify this issue by presenting layerdependent and polarization-resolved Raman studies of $\mathrm{ReS}_{2}$ in the ultralow frequency regime $\left(<50 \mathrm{~cm}^{-1}\right)$. We clearly observe the LSM and LBM for bilayers and thicker samples. The layer-dependent peak positions enable a reliable determination of the thickness of $\mathrm{ReS}_{2}$ by Raman spectroscopy. Angle-dependent measurements demonstrate the anisotropic Raman intensity of the interlayer modes and reveal a lifting of the degeneracy of the LSM, which is expected for crystals with anisotropic shear modulus.

Our sample preparation starts with mechanical exfoliation of $\mathrm{ReS}_{2}$ bulk crystals (source: 2D Semiconductors Inc.) onto a polydimethylsiloxane (PDMS) stamp. Relevant flakes are identified using an optical microscope and subsequently transferred on a p-doped Si-Chip with a $285 \mathrm{~nm} \mathrm{SiO}_{2}$ capping layer by applying a recently developed all-dry transfer technique ${ }^{22}$. None of the samples showed indications of degradation when stored in ambient conditions for several weeks. Raman measurements of the samples are carried out at room temperature in backscattering geometry with a linearly polarized $532 \mathrm{~nm} \mathrm{cw}$ laser which is coupled into a $100 \mathrm{x}$ microscope objective (spot size $1 \mu \mathrm{m}$ ), further experimental details are published elsewhere 23 . All Raman measurements are performed in cross-polarized geometry to suppress the spectrally broad background of inelastically scattered light which stems from free carriers in the heavily p-doped Si substrate ${ }^{24}$. To access the ultralow frequency regime we use three reflective volume Bragg grating filters which are placed in front of the spectrometer. Careful adjustment of the setup allows the simultaneous observation of Stokes and Anti-Stokes Raman signals and detection of Raman peaks down to $5 \mathrm{~cm}^{-1}$. For angleresolved Raman measurements we rotate the sample on 

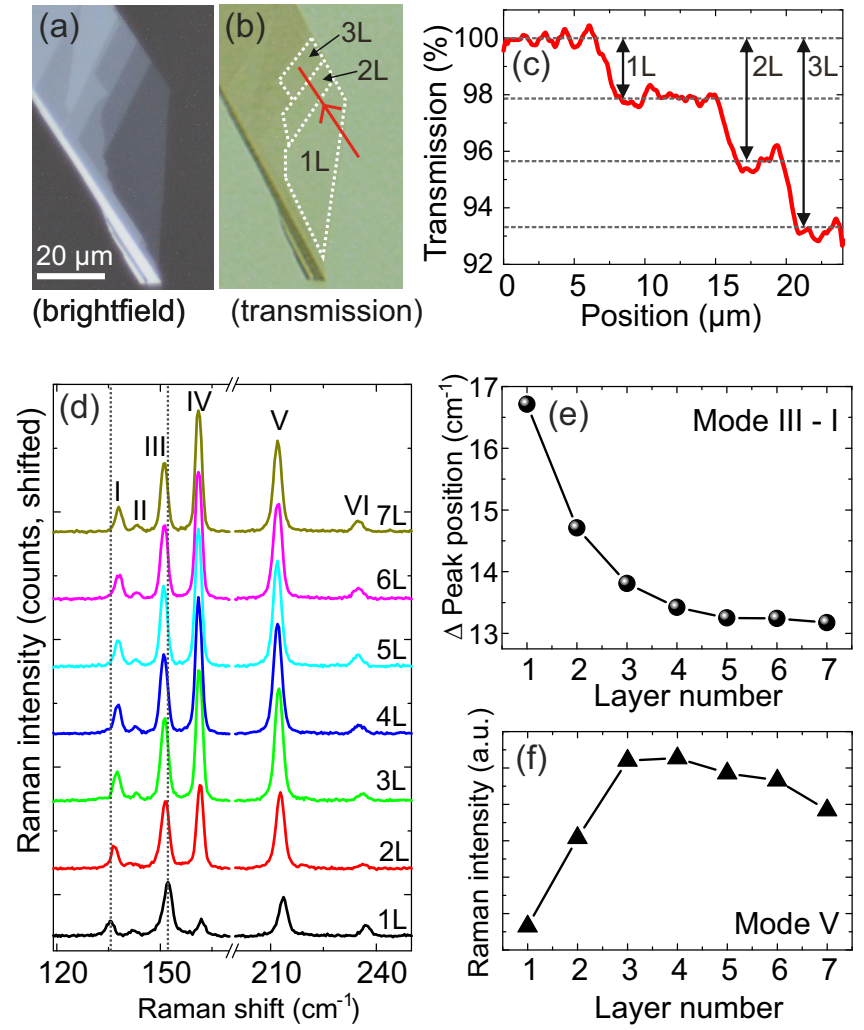

FIG. 1. (a) Brightfield and (b) transmission optical microscope images of a $\mathrm{ReS}_{2}$ flake on a PDMS substrate. The dotted lines in (b) highlight areas of different thickness. The red arrow indicates the position of the linescan shown in (c). (c) Normalized transmission trace extracted from the red channel of (b). (d) Layer-dependent high-energy Raman spectra of $\mathrm{ReS}_{2}$. The dotted lines indicate the peak positions of mode I and III, respectively, in the ML case. (e) Peak frequency difference of mode III and mode I in dependence of the layer number. (f) Raman intensity of mode $\mathrm{V}$ in dependence of the layer number.

a rotary stage while keeping the linear polarization of the laser and the orientation of the polarizer in front of the spectrometer fixed.

First, we establish a basic understanding of the layer thickness of our samples. Fig. 11(a) shows a brightfield image of a $\mathrm{ReS}_{2}$ flake which has been mechanically exfoliated on a PDMS stamp. Terraces of different thickness of the crystal can be identified but no clear statement about the absolute layer thickness can be made from these brightfield images. However, it has been shown that the absorption of atomically thin materials is fundamentally related to the fine structure constant with one layer absorbing around $2 \%$ of the incident light ${ }^{25 \mid 26}$. Therefore, we examine the same flake in a transmission microscope (Fig. 1(b)) and extract the absolute amount of absorbed light from the red channel of the image (Fig. 1(c)). The well-defined steps in the transmission of the flake with values of around $2 \%$ for each individual terrace demonstrate that the sample is indeed atomically thin with areas down to the ML limit.

Furthermore, recent Raman measurements have shown that, in analogy to $\mathrm{MoS}_{2} 27$, the energetic difference of certain high-energy modes of $\mathrm{ReS}_{2}$ can be used to assign the thickness of $\mathrm{ReS}_{2}$ flakes from one layer up to four layers $\frac{15}{15}$. Fig. 1(d) displays the layer-dependent high-energy Raman modes of $\mathrm{ReS}_{2}$ up to 7 layers. In line with recent literature $4 \mid 415$ we label the individual peaks with Roman numerals from I to VI. For the respective peak positions in the case of a ML we measure: $135.5 \mathrm{~cm}^{-1}, 142.2 \mathrm{~cm}^{-1}, 152.2 \mathrm{~cm}^{-1}, 161.9 \mathrm{~cm}^{-1}$, $213.6 \mathrm{~cm}^{-1}$ and $237.1 \mathrm{~cm}^{-1}$, which is in good agreement with references ${ }^{4}$ and ${ }^{15}$. Mode I and mode II are assigned to $\mathrm{A}_{g^{-}}$-like vibrational modes, while modes III-VI are $\mathrm{E}_{g^{-}}$ like vibrational modes ${ }^{4}$. The peak position of mode I increases from $135.5 \mathrm{~cm}^{-1}$ in the ML to $137.6 \mathrm{~cm}^{-1}$ for a $4 \mathrm{~L}$ sample. By contrast, the position of mode III decreases from $152.2 \mathrm{~cm}^{-1}$ in the ML to $151.1 \mathrm{~cm}^{-1}$ for a $4 \mathrm{~L}$ sample. Hence, the difference of the peak positions of mode III and mode I provides a clear indication for the thickness of $\mathrm{ReS}_{2}$ up to four layers (Fig. 1(e)) ranging from $16.7 \mathrm{~cm}^{-1}$ in the ML to $13.4 \mathrm{~cm}^{-1}$ in the $4 \mathrm{~L}$. However, from five layers on, the peak difference converges around $13.2 \mathrm{~cm}^{-1}$ and is therefore no longer an unambiguous measure for the layer thickness. We also determine the absolute Raman signal for each mode with respect to the layer thickness. Figure 1(f) shows the Raman intensity of mode $\mathrm{V}$ from $1 \mathrm{~L}$ to $7 \mathrm{~L}$. One can observe that the absolute Raman signal increases from $1 \mathrm{~L}$ on and reaches its maximum in the region of $3 \mathrm{~L}$ to $4 \mathrm{~L}$. This layer-dependent intensity behavior was also observed for the closely related $\operatorname{ReSe}_{2}{ }^{28}$ and further supports our assignment. This effect can be understood by taking into account the optical interference effects between the atomically thin material and the $\mathrm{SiO}_{2} / \mathrm{Si}$-substrate which lead to a maximum in the enhancement factor for three layers 29 .

We now turn to the interlayer Raman modes, which are found in the ultralow frequency regime. Generally, for an N-layered crystal there are N-1 LBMs perpendicular to the sample plane and N-1 LSMs along the crystal plane ${ }^{30}$. In isotropic crystals, such as graphene or $\mathrm{MoS}_{2}$, the LSMs are doubly degenerate. It is expected, however, that this degeneracy is lifted in materials with in-plane anisotropy 31 . The frequencies of the LBM and LSM as a function of $\mathrm{N}$ can be well-described using a monoatomic chain model 18 . Thereby, the individual MLs are considered as single mass units which are held together by weak interlayer bonds. This model yields a very simple result for the highest- $\left(\omega_{+}\right)$and lowest-energy $\left(\omega_{-}\right)$LBM and LSM for a given number of layers:

$$
\omega_{ \pm}(N)=\omega(2) \sqrt{1 \pm \cos \left(\frac{\pi}{N}\right)},
$$

where $\omega(2)$ are the frequencies of the LBM and LSM for $2 \mathrm{~L}$, respectively.

Figure 2(a) shows the layer-dependent Raman spectra of $\mathrm{ReS}_{2}$ ranging from $1 \mathrm{~L}$ to $10 \mathrm{~L}$ close to the laser line, 
which were obtained on the sample shown in Fig. 2(b). For these measurements, a cleaved edge of the sample was aligned parallel to the linear polarization of the laser, corresponding to a rotation angle of $\theta=0^{\circ}$ (see Fig. 2(b)). As expected for any layered material we do not observe
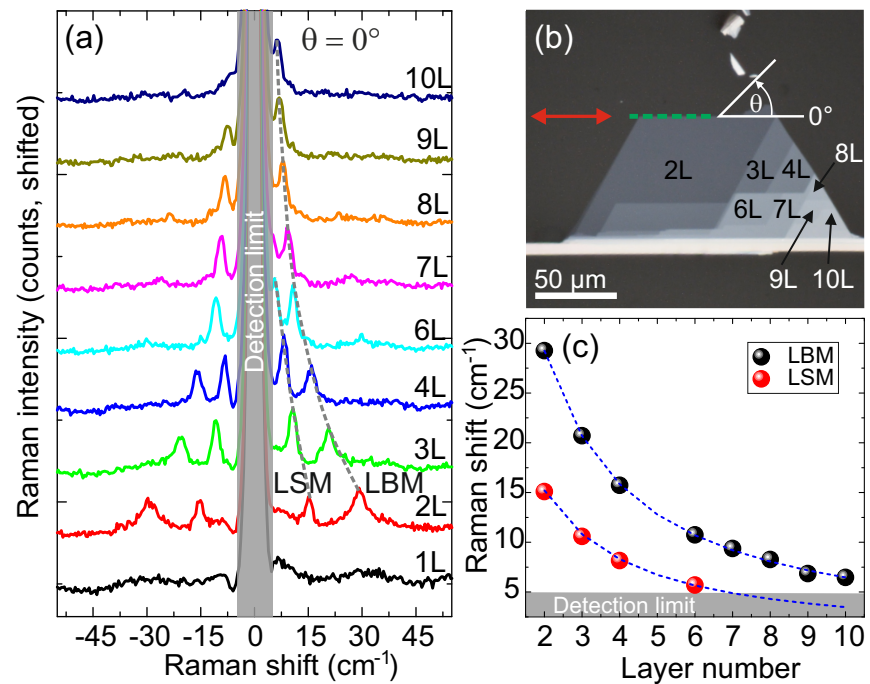

FIG. 2. (a) Layer series of Raman spectra of $\mathrm{ReS}_{2}$ in the ultralow frequency regime. The dotted lines are guides to the eye. (b) Brightfield image of the few-layer $\mathrm{ReS}_{2}$-flake (on the PDMS film before transfer) on which the spectra shown in (a) were obtained. The red double arrow shows the orientation of the linearly polarized laser. The green dotted line defines the cleaved edge of the crystal. The white angle shows the direction of rotation and the definition of the angle $\theta$. (c) Layer-dependent peak positions of LBM (black spheres) and LSM (red spheres). The blue dotted lines are fit functions which result from the applied monoatomic chain model.

interlayer modes in the ML. In the case of a bilayer we identify two features at $15.1 \mathrm{~cm}^{-1}$ and $29.3 \mathrm{~cm}^{-1}$, which we relate to the LSM and LBM, respectively. Angleresolved Raman measurements of both modes will later confirm this assignment. The LSM and LBM apparently possess a considerable coupling strength which renders their relative intensity comparable to the features of the high-energy Raman modes (see Fig. 1 of the supporting information). For all layers, we observe only the energetically lowest $\left(\omega_{-}\right)$modes of the LSM and LBM, albeit N-1 modes are theoretically allowed. This observation might be related to a vanishing electron-phonon coupling strength of the higher-lying modes. In contrast to, e.g., $\mathrm{MoS}_{2}$, where the LBM is suppressed in cross-polarized backscattering geometry due to symmetry reasons ${ }^{18}$, we clearly observe it in $\mathrm{ReS}_{2}$ with a similar amplitude as the LSM.

Figure 2(c) shows the peak position of both LSM and LBM with respect to the number of layers. The values were obtained by using Lorentz-shaped fit functions. Both modes show a softening with increasing $\mathrm{N}$, the LBM from $29.3 \mathrm{~cm}^{-1}$ in $2 \mathrm{~L}$ to $6.5 \mathrm{~cm}^{-1}$ in $10 \mathrm{~L}$ and the LSM from $15.1 \mathrm{~cm}^{-1}$ in $2 \mathrm{~L}$ to $5.7 \mathrm{~cm}^{-1}$ in $6 \mathrm{~L}$. For larger $\mathrm{N}$, the LSM shifts below the detection limit. While this behavior is typically observed for the LBM in other layered materials, we point out here that only a few reports exist of the softening branch of the LSM 18131 . By contrast, the most prominent LSM in graphene $e^{16}$ and $\mathrm{MoS}_{2}{ }^{17 / 18}$ is the $\omega_{+}$mode that stiffens with increasing $\mathrm{N}$. This stiffening branch of the LSM is not visible in our measurements, which is in line with a recent study on the closely related $\mathrm{ReSe}_{2}{ }_{31}$. In order to fit the extracted peak positions of LBM and LSM we resort to the monoatomic chain model described above, using the layer dependence of the lowest-energy modes $\omega_{-}$. As can be seen in Fig. 2(c) the model quantitatively describes the peak positions of both interlayer modes. We therefore conclude that the peak positions of the interlayer modes present an unambiguous and destruction-free method to determine the thickness of $\mathrm{ReS}_{2}$ for up to ten layers.

Finally, we present our results of angle-resolved Raman measurements of $\mathrm{ReS}_{2}$ in the ultralow frequency range. As discussed above, we define the rotation angle $\theta=0^{\circ}$ for the incident laser polarization being aligned parallel to the cleaved edge of our sample indicated in Fig. 2(b). It was suggested recently that well-defined edges of mechanically exfoliated $\mathrm{ReS}_{2}$ often represent the $b$-axis of the crystal 15 . Thereby it was also shown that the respective cleaved edge lies exactly between the intensity lobes of the high-energetic mode $\mathrm{V}$ in cross-polarization geometry. Comparing this observation with our results, Fig. 3(a) shows the polarization-resolved Raman measurements of mode $\mathrm{V}$ in $4 \mathrm{~L}-\mathrm{ReS}_{2}$. Since the cleaved edge of the crystal lies also exactly between the main lobes of the signal we assign this direction to the crystal $b$-axis. Figure 3(b) and (c) display the angle-resolved Raman measurements of the LBM and LSM in $4 \mathrm{~L} \mathrm{ReS}_{2}$. Both modes clearly exhibit an anisotropic behavior with four intensity maxima which is a direct consequence of the crystal anisotropy of $\mathrm{ReS}_{2}$. In the case of the LBM the maxima at $0^{\circ}$ and $180^{\circ}$ lie directly parallel to the cleaved edge of the sample which can serve as an additional fingerprint for the crystal orientation of $\mathrm{ReS}_{2}$. We note that the angles of maximum intensity of the LSM are shifted by $20^{\circ}$ with respect to the LBM, which highlights the different nature of these two low-energetic features.

Furthermore, it is also expected that the energetic positions of LBM and LSM show a differing behavior in angleresolved measurements. Since $\mathrm{ReS}_{2}$ possesses an in-plane anisotropy, the degeneracy of the LSM energy should be lifted as it represents a rigid in-plane vibration. The energy of the LBM however, being an out-of-plane vibration, should naturally not be affected by the in-plane anisotropy. Figure 3(d) displays the measured peak position of LSM and LBM for $4 \mathrm{~L} \mathrm{ReS}_{2}$ in dependence of the rotation angle. The energy of the LSM shows a periodic oscillation with a minimum around $7.8 \pm 0.3 \mathrm{~cm}^{-1}$ and a maximum of $8.4 \pm 0.3 \mathrm{~cm}^{-1}$ yielding an energetic shift of about $0.6 \pm 0.3 \mathrm{~cm}^{-1}$. This angle-dependent shift indicates that the interlayer shear modulus is anisotropic. Using the monoatomic chain mode ${ }^{[16] 18}$, we can estimate 

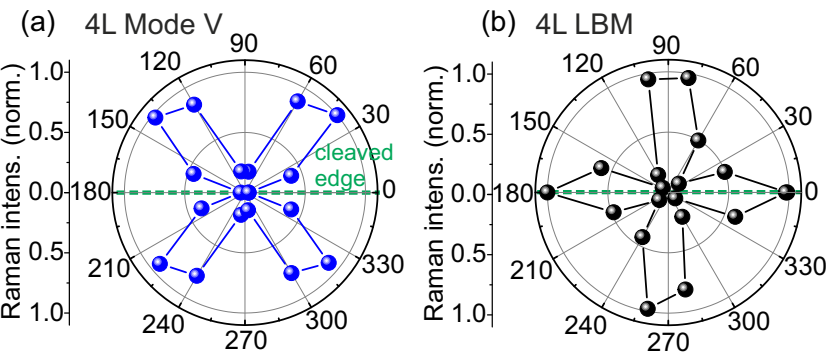

(c) 4L LSM
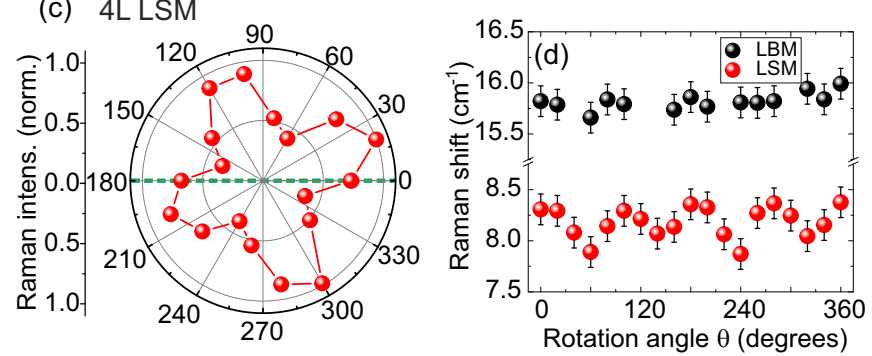

FIG. 3. (a) Angle-resolved Raman peak intensity of the mode $\mathrm{V}$ in $4 \mathrm{~L} \mathrm{ReS}$ located at $212.0 \mathrm{~cm}^{-1}$. (b) Angle-resolved Raman peak intensity of the LBM in $4 \mathrm{~L} \mathrm{ReS}_{2}$. (c) Angle-resolved Raman peak intensity of the LSM in $4 \mathrm{~L} \mathrm{ReS}_{2}$. The maximum of intensity is shifted by $20^{\circ}$ with respect to the LBM. The cleaved edge of the sample defined in Fig. 2(b) is marked by the green dotted lines in (a)-(c). The peak intensities shown in (a)-(c) are normalized to the maximum Raman peak intensity of the respective modes. (d) Peak positions of LBM (black spheres) and LSM (red spheres) as a function of the rotation angle. The error bars indicate the spectral resolution of the setup.

that this anisotropy is on the order of $14 \%$. Recently, a theoretical calculation predicted a splitting of the LSM in the structurally closely related $\mathrm{ReSe}_{2}$ in the order of $1 \mathrm{~cm}^{-131}$ which is in good agreement with our obtained value for $\mathrm{ReS}_{2}$. In contrast to the LSM, the energy of the LBM does not exhibit any systematic variation with the rotation angle. This observation corroborates our prior spectral assignment of the two peaks. We also note that none of the high-energy Raman modes show a systematic frequency shift with the rotation angle (see Fig. 2 of the supporting information), in agreement with the study by Chenet et al $\frac{15}{15}$, demonstrating the unique sensitivity of the LSM to interlayer coupling anisotropy.

In conclusion, we have presented Raman measurements of $\mathrm{ReS}_{2}$ in the ultralow frequency range. Thereby, we were able to identify and track layer-dependent features of the LBM and the LSM which stem from the interlayer interaction of the crystal. The presence of these modes indicates that $\mathrm{ReS}_{2}$ is isostructural to $\mathrm{ReSe}_{2}$. The layer dependence of their peak positions can be readily reproduced by means of a monoatomic chain model and provide an easy and non-destructive method for determining the layer number of $\mathrm{ReS}_{2}$ up to 10L. Angle-resolved Raman measurements clearly confirm the anisotropic nature of both interlayer modes. By tracing the peak position of the LSM with respect to the polarization plane we are able to unveil an energetic shift of about $0.6 \mathrm{~cm}^{-1}$ which is directly related to the in-plane shear modulus anisotropy of the crystal. Our Raman measurements on $\mathrm{ReS}_{2}$ therefore should foster the understanding of the fascinating anisotropic properties of this novel 2D material.

\section{ACKNOWLEDGEMENTS}

The authors ackowledge financial support by the DFG via KO3612/1-1, GRK1570 and SFB689.
* tobias.korn@physik.uni-regensburg.de

1 S. Tongay, H. Sahin, C. Ko, A. Luce, W. Fan, K. Liu, J. Zhou, Y.-S. Huang, C.-H. Ho, J. Yan, D. F. Ogletree, S. Aloni, J. Ji, S. Li, J. Li, F. M. Peeters, and J. Wu, Nat. Commun. 5, 3252 (2014), arXiv:1510.02128

${ }^{2}$ K. S. Novoselov, D. Jiang, F. Schedin, T. J. Booth, V. V. Khotkevich, S. V. Morozov, and A. K. Geim, Proc. Natl. Acad. Sci. 102, 10451 (2005).

${ }^{3}$ S. Horzum, D. Çakr, J. Suh, S. Tongay, Y.-S. Huang, C.-H. Ho, J. Wu, H. Sahin, and F. M. Peeters, Phys. Rev. B 89, 155433 (2014)

${ }^{4}$ Y. Feng, W. Zhou, Y. Wang, J. Zhou, E. Liu, Y. Fu, Z. Ni, X. Wu, H. Yuan, F. Miao, B. Wang, X. Wan, and D. Xing, Phys. Rev. B 92, 054110 (2015)

5 K. Friemelt, M.-C. Lux-Steiner, and E. Bucher, J. Appl. Phys. 74, 5266 (1993)

${ }^{5}$ J.-L. Bobet, B. Chevalier, and D. Fruchart, Solid State Phenom. 170, 135 (2011).

7 C. M. Corbet, C. McClellan, A. Rai, S. S. Sonde, E. Tutuc, and S. K. Banerjee, ACS Nano 9, 363 (2015).
8 Y.-C. Lin, H.-P. Komsa, C.-H. Yeh, T. Björkman, Z.Y. Liang, C.-H. Ho, Y.-S. Huang, P.-W. Chiu, A. V. Krasheninnikov, and K. Suenaga, ACS Nano (2015), 10.1021/acsnano.5b04851

${ }^{9}$ C. H. Lui, Z. Ye, C. Ji, K.-C. Chiu, C.-T. Chou, T. I. Andersen, C. Means-Shively, H. Anderson, J.-M. Wu, T. Kidd, Y.-H. Lee, and R. He, Phys. Rev. B 91, 1 (2015).

10 E. Liu, Y. Fu, Y. Wang, Y. Feng, H. Liu, X. Wan, W. Zhou, B. Wang, L. Shao, C.-H. Ho, Y.-S. Huang, Z. Cao, L. Wang, A. Li, J. Zeng, F. Song, X. Wang, Y. Shi, H. Yuan, H. Y. Hwang, Y. Cui, F. Miao, and D. Xing, Nat. Commun. 6, 6991 (2015)

11 T. Fujita, Y. Ito, Y. Tan, H. Yamaguchi, D. Hojo, A. Hirata, D. Voiry, M. Chhowalla, and M. Chen, Nanoscale 6, 12458 (2014)

${ }^{12}$ K. Keyshar, Y. Gong, G. Ye, G. Brunetto, W. Zhou, D. P. Cole, K. Hackenberg, Y. He, L. Machado, M. Kabbani, A. H. C. Hart, B. Li, D. S. Galvao, A. George, R. Vajtai, C. S. Tiwary, and P. M. Ajayan, Adv. Mater. 27, 4640 (2015) 
13 A. C. Ferrari and D. M. Basko, Nat. Nanotechnol. 8, 235 (2013) arXiv:arXiv:1306.5856v1.

${ }^{14}$ X. Zhang, X.-F. Qiao, W. Shi, J.-B. Wu, D.-S. Jiang, and P.-H. Tan, Chem. Soc. Rev. 44, 2757 (2015) arXiv:arXiv:1502.00701v1

15 D. A. Chenet, O. B. Aslan, P. Y. Huang, C. Fan, A. M. van der Zande, T. F. Heinz, and J. C. Hone, Nano Lett. 15, 5667 (2015)

${ }^{10}$ P. H. Tan, W. P. Han, W. J. Zhao, Z. H. Wu, K. Chang, H. Wang, Y. F. Wang, N. Bonini, N. Marzari, N. Pugno, G. Savini, A. Lombardo, and A. C. Ferrari, Nat. Mater. 11, 294 (2012)

Ir G. Plechinger, S. Heydrich, J. Eroms, D. Weiss, C. Schüller, and T. Korn, Appl. Phys. Lett. 101, 101906 (2012) arXiv:1205.1916

18 X. Zhang, W. P. Han, J. B. Wu, S. Milana, Y. Lu, Q. Q. Li, A. C. Ferrari, and P. H. Tan, Phys. Rev. B 87, 1 (2012), arXiv:1212.6796

19 Y. Zhao, X. Luo, H. Li, J. Zhang, P. T. Araujo, C. K. Gan, J. Wu, H. Zhang, S. Y. Quek, M. S. Dresselhaus, and Q. Xiong, Nano Lett. 13, 1007 (2013)

20 S.-Y. Chen, C. Zheng, M. S. Fuhrer, and J. Yan, Nano Lett. 15, 2526 (2015)

${ }_{21}$ G. Froehlicher, E. Lorchat, F. Fernique, C. Joshi, A. Molina-Sánchez, L. Wirtz, and S. Berciaud, Nano Lett.
15, $6481(2015)$

$\overline{22}$ A. Castellanos-Gomez, M. Buscema, R. Molenaar, V. Singh, L. Janssen, H. S. J. van der Zant, and G. A. Steele, 2D Mater. 1, 011002 (2014), arXiv:1311.4829

23 G. Plechinger, F. Mooshammer, A. Castellanos-Gomez, G. A. Steele, C. Schüller, and T. Korn, 2D Materials 2, 034016 (2015)

${ }^{24}$ M. Chandrasekhar, U. Rössler, and M. Cardona, Phys. Rev. B 22, 761 (1980)

${ }^{25}$ R. R. Nair, P. Blake, A. N. Grigorenko, K. S. Novoselov, T. J. Booth, T. Stauber, N. M. R. Peres, and A. K. Geim, Sciene 320, 1308 (2008), arXiv:0803.3718v1

26 H. Fang, H. A. Bechtel, E. Plis, M. C. Martin, S. Krishna, E. Yablonovitch, and A. Javey, Proc. Natl. Acad. Sci. 110, 11688 (2013).

${ }^{2}$ C. Lee, H. Yan, L. E. Brus, T. F. Heinz, J. Hone, and S. Ryu, ACS Nano 4, 2695 (2010), arXiv:1005.2509

28 D. Wolverson, S. Crampin, A. S. Kazemi, A. Ilie, and S. J. Bending, ACS Nano 8, 11154 (2014).

29 S.-L. Li, H. Miyazaki, H. Song, H. Kuramochi, S. Nakaharai, and K. Tsukagoshi, ACS Nano 6, 7381 (2012).

${ }^{30}$ K. H. Michel and B. Verberck, Phys. Rev. B 85, 1 (2012).

31 H. Zhao, J. Wu, H. Zhong, Q. Guo, X. Wang, F. Xia, L. Yang, P. Tan, and H. Wang, Nano Research 8, 3651 (2015)

\section{Supplementary note 1: direct comparison of low- and high-energy Raman modes}

The LSM and LBM have Raman intensities that are on the same order of magnitude as the higher-energy modes. Figure 4 demonstrates this with a Raman spectrum obtained on $4 \mathrm{~L} \mathrm{ReS}_{2}$.

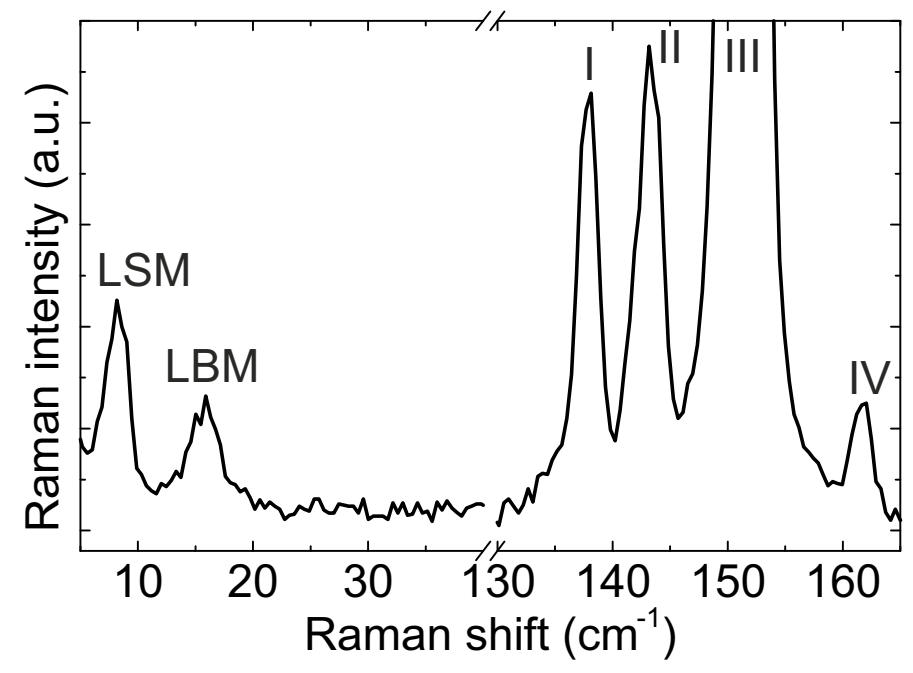

FIG. 4. Raman spectrum of $4 \mathrm{~L} \mathrm{ReS}_{2}$ showing the low-energy region relevant for LBM and LSM, as well as the high-energy region containing modes I-IV. The y axis is the same for the two regions.

\section{Supplementary note 2: Mode frequencies of higher-energy modes as a function of angle}

None of the high-energy Raman modes in $\mathrm{ReS}_{2}$ show a systematic frequency shift with the rotation angle, in contrast to the LSM (Fig. 3(d) in the main text). Figure 4 demonstrates this for the high-energy modes I-V in $4 \mathrm{~L}$ ReS 2 . The mode position values were obtained by using Lorentz-shaped fit functions. 

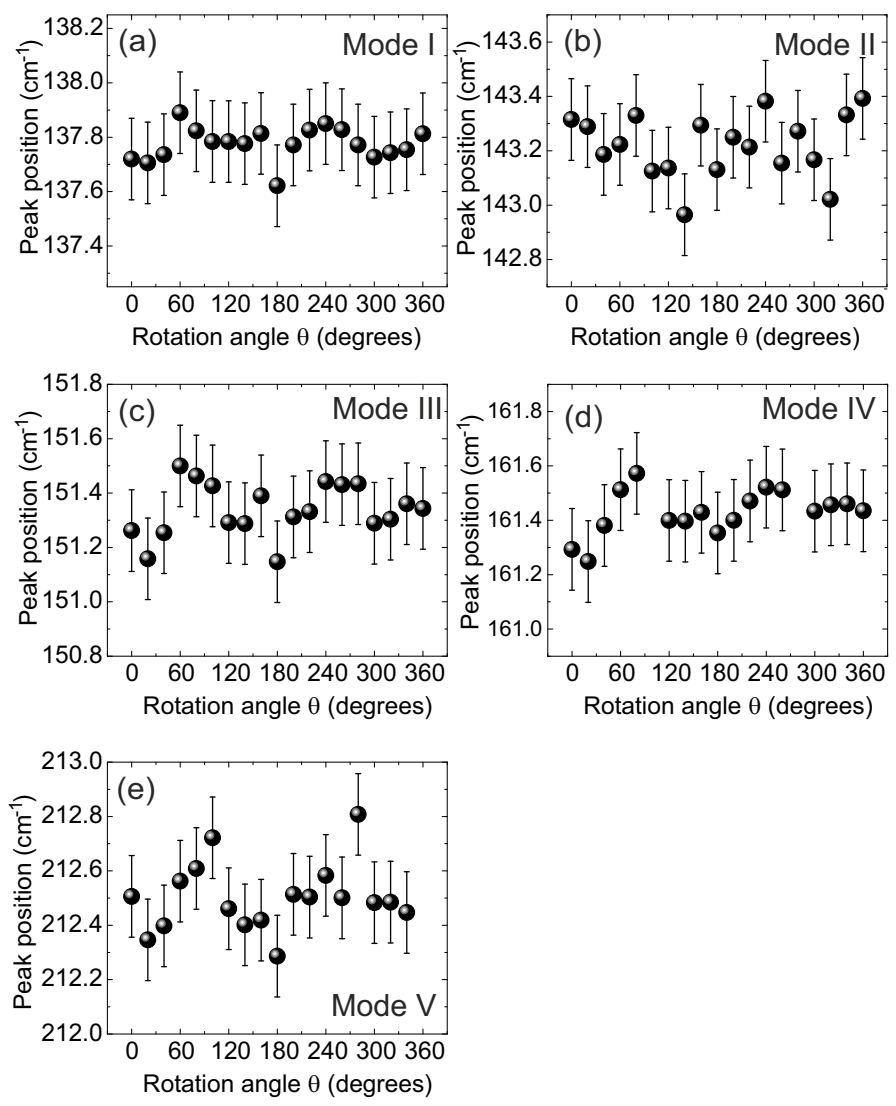

FIG. 5. (a)-(e) Mode frequencies of the high-energy modes I-V extracted from angle-resolved Raman measurements on $4 \mathrm{~L}$ $\mathrm{ReS}_{2}$. The y axis covers the same frequency range for all graphs. 